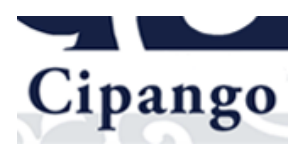

\title{
Cipango
}

Cahiers d'études japonaises

16 | 2009

L'invention des « arts populaires » - Yanagi Sōetsu et le Mingei

\section{Des « nippologies » dans les musées}

Design et identité japonaise à Paris

"Nihonjinron" in the design and Japanese identity museums of Paris

\section{Coralie Castel}

\section{(2) OpenEdition \\ Journals}

\section{Édition électronique}

URL : https://journals.openedition.org/cipango/389

DOI : $10.4000 /$ cipango.389

ISSN : 2260-7706

\section{Éditeur}

INALCO

Édition imprimée

Date de publication : 1 janvier 2009

Pagination : 121-147

ISSN : 1164-5857

\section{Référence électronique}

Coralie Castel, «Des «nippologies » dans les musées », Cipango [En ligne], 16 | 2009, mis en ligne le 18 novembre 2011, consulté le 30 juin 2021. URL : http://journals.openedition.org/cipango/389; DOI : https://doi.org/10.4000/cipango.389

Ce document a été généré automatiquement le 30 juin 2021.

\section{(c) (i) \&}

Cipango est mis à disposition selon les termes de la Licence Creative Commons Attribution - Pas d'Utilisation Commerciale 4.0 International. 


\title{
Des « nippologies » dans les musées
}

\author{
Design et identité japonaise à Paris \\ "Nihonjinron" in the design and Japanese identity museums of Paris
}

Coralie Castel

1 Trois expositions sur le design japonais se sont tenues à Paris récemment, dans le cadre de la commémoration du cent-cinquantième anniversaire de l'établissement des relations diplomatiques entre la France et le Japon. Du 30 septembre 2008 au 11 janvier 2009, le Musée du quai Branly a présenté L'esprit mingei au Japon: de l'artisanat populaire au design. Du 22 octobre 2008 au 31 janvier 2009, la Maison de la culture du Japon à Paris (ci-après MCJP) proposait Wa : l'harmonie au quotidien, design japonais d'aujourd'hui. Enfin, du 12 au 21 décembre 2008, le Musée des Arts décoratifs a accueilli l'exposition Kansei: Japan design exhibition.

2 Ces trois expositions ont été organisées de façon indépendante, sans concertation entre les trois établissements. L'exposition du Musée du quai Branly a été conçue en collaboration avec le Musée des arts populaires de Tōkyō ou Mingeikan. Celle de la MCJP a été élaborée par le siège de la Fondation du Japon à Tōkyō. Enfin, l'exposition des Arts décoratifs a été entièrement montée par le ministère japonais de l'Économie, du Commerce et de l'Industrie (METI) et l'Organisation japonaise du commerce extérieur (JETRO). Ainsi, ces trois expositions ont été réalisées, en partie pour la première, et presque entièrement pour les deux dernières, par des Japonais.

Le sociologue Jean Davallon, dans L'exposition à l'œuvre ${ }^{1}$, affirme que l'élaboration d'une exposition relève d'un propos construit, et qu'elle est sous-tendue par une vision du monde. En tant que média, elle ne fait pas que montrer des objets : elle indique aussi, et surtout, comment regarder ces objets. Elle obéit en cela à des "stratégies communicationnelles ». La même idée est à la base de la réflexion de l'anthropologue Benoît de l'Estoile qui retrace dans Le goût des autres l'histoire des «musées des autres » en France ${ }^{2}$ :

Parce que la mise en exposition est la traduction spatiale et sensorielle (en particulier visuelle) de conceptions explicites ou implicites, elle offre une matérialisation de visions $d u$ monde. Le musée est à la fois l'expression de courants artistiques, scientifiques, politiques qui se développent en dehors de lui et un lieu de production et de diffusion de 
représentations, qui contribuent pour une part non négligeable à définir la réalité et à constituer les cadres d'interprétation à partir desquels celle-ci est déchiffrée.

En m'appuyant sur ce constat, je me suis donnée pour tâche d'observer la façon dont les trois expositions ci-dessus ont représenté le Japon, alors même qu'elles se sont tenues dans trois institutions distinctes, dont les ambitions de dialogue interculturel, de promotion de la culture japonaise, ou encore commerciales, sont différentes. Plus concrètement, je souhaite m'interroger sur la nature des représentations du Japon dans le monde qui ont été exposées, à Paris, pendant l'hiver 2008.

5 L'analyse ethnographique de ces expositions s'est d'abord portée sur l'observation attentive des lieux. Les objets, la scénographie, les panneaux explicatifs fournissent des informations au visiteur; mais plus encore, brochures, plans et autres supports écrits distribués au public explicitent le propos. Les discours recueillis auprès des organisateurs, côté français, permettent également de mettre en lumière certains partis pris de la mise en exposition. Enfin, les communiqués de presse envoyés par les institutions organisatrices, et, en retour, l'écho de ces expositions dans la presse, permettent d'évaluer la façon dont leurs messages ont été perçus et relayés.

6 Au sein de chaque exposition, l'observation révèle le caractère hétéroclite des objets présentés : la coexistence d'objets de registres variés suscite un sentiment de confusion, renforcé par des scénographies assez discrètes. Pourtant, il ressort toujours "un » Japon présenté comme homogène. Quels sont les leitmotivs qui permettent d'établir la cohérence identitaire de ces expositions ? Relèvent-ils d'un mode de discours récurrent sur le Japon, et du Japon sur lui-même?

\section{Le Musée du quai Branly et l'« esprit mingei »}

7 Le Musée du quai Branly vise à présenter les cultures du monde dans leur diversité, à travers des expositions qui accordent une large part à l'appréciation esthétique des objets exposés.

8 L'esprit mingei au Japon: de l'artisanat populaire au design se focalise sur la personne de Yanagi Sōetsu 柳宗悦 (1889-1961) et sur son mouvement de découverte et de mise en valeur des artisanats japonais et coréen initié à partir des années 1920. L'exposition a été réalisée sous la direction de Germain Viatte, commissaire d'exposition au Musée du quai Branly, mais à partir d'objets provenant presque tous du Mingeikan, le musée fondé par Yanagi en 1936.

9 La scénographie de la salle est très sommaire. Le fond des vitrines est blanc, et les objets sont posés sur des supports neutres. Aux murs, quelques cartels résument la démarche de Yanagi Sōetsu. L'ensemble, très neutre, ne laisse pas voir de parti pris spécifique au Musée du quai Branly par rapport au Mingeikan. 
Une vitrine de l'exposition Mingei, Musée du quai Branly, 2008.

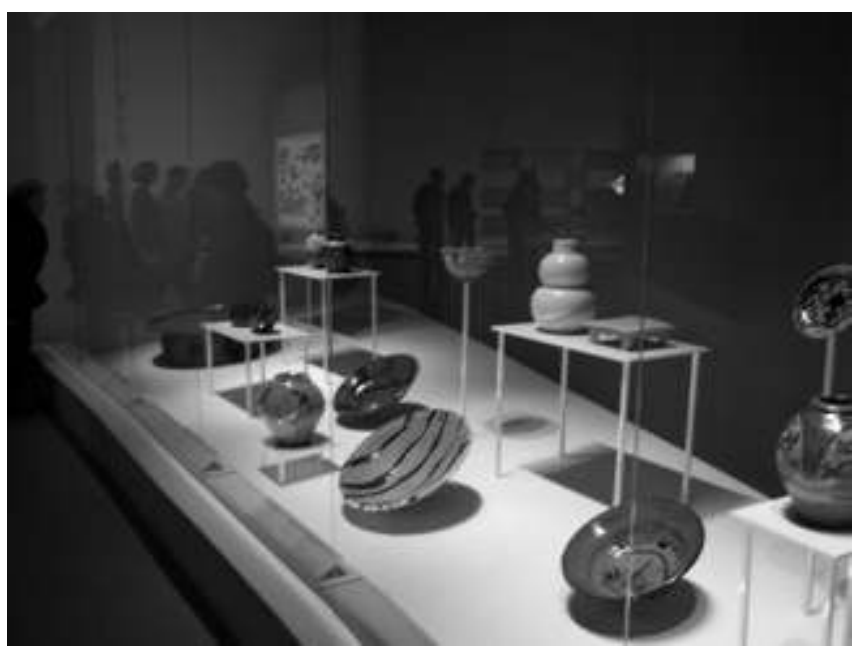

(PHOTO DE L'AUTEUR)

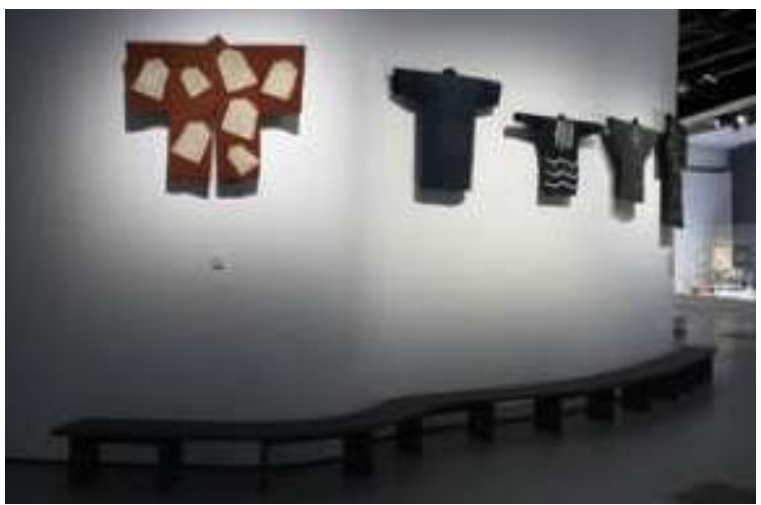

Les vêtements présentés dans l'exposition Mingei sont mis en valeur pour leur esthétique. Un simple carton donne leur date de fabrication approximative, mais aucune autre information n'est fournie. Musée du quai Branly, 2008.

(PHOTO DE L'AUTEUR)

Les objets de la première vitrine sont de provenances géographiques et d'époques très variées : un plat créé en 1952 par le potier anglais Bernard Leach, qui a vécu au Japon, côtoie des bols et des plateaux chinois ou coréens datés $\mathrm{du}_{\mathrm{XVI}}{ }^{\mathrm{e}}$ au $\mathrm{XIX}^{\mathrm{e}}$ siècle, une sculpture de l'époque Jōmon ( $\mathrm{du} \mathrm{x}^{\mathrm{e}}$ siècle avant $\mathrm{J}$.-C.) ou encore une urne funéraire d'Okinawa datée $\mathrm{du} \mathrm{xx}^{\mathrm{e}}$ siècle.

11 La vitrine suivante regroupe trois ensembles : «Les arts populaires au Japon » présente des laques, des poteries, des vêtements du Japon datant des XVIII $^{\mathrm{e}}$ et $\mathrm{xIX}^{\mathrm{e}}$ siècles; quelques pas plus loin, «Les arts populaires à la périphérie de l'archipel» expose des objets coréens et d'Okinawa fabriqués entre le $\mathrm{xvI}^{\mathrm{e}}$ et le $\mathrm{xx}^{\mathrm{e}}$ siècle ; enfin, «Les premiers complices de Yanagi Sōetsu » montre des poteries réalisées dans les années 193019 à 1940 par des artistes influencés par le mingei.

12 Une grande salle ronde au fond de la galerie d'exposition est consacrée à "Trois créateurs internationaux au Japon » ayant œuvré entre 1930 et 1950 : l'Allemand Bruno Taut (laques), l'Américain Isamu Noguchi (luminaires, meubles, terres cuites), et la Française Charlotte Perriand (mobilier). L'espace "Yanagi Sōri et la question du design » est réservé aux créations les plus célèbres du designer, fils de Sōetsu, réalisées 
au cours des années 1950 à 1970 : le tabouret Butterfly, créé en 1956, est mis en valeur dans une vitrine séparée. Sur le chemin de la sortie, une table basse rassemble les «objets en bambou » : des objets anonymes de l'époque d'Edo y côtoient des créations de Bruno Taut.

De façon générale, et jusqu'à l'intérieur des vitrines, l'exposition montre une juxtaposition d'objets d'origines et d'époques très diverses. Ils répondent aussi à des registres différents: la présence des objets anonymes procède d'une démarche de conservation tandis que d'autres, dont les auteurs sont mis en avant, sont issus d'une démarche de création.

\section{« L'harmonie au quotidien » à la Maison de la culture du Japon}

La Maison de la culture du Japon à Paris, en tant qu'institution, dépend pour partie de la Fondation du Japon dont le siège est à Tōkyō. Elle organise régulièrement des expositions sur des thèmes variés, conformément à sa mission de promotion de la culture japonaise.

L'exposition Wa: l'harmonie au quotidien, design japonais d'aujourd'hui propose des objets très différents de ceux du quai Branly. Le visiteur entre par un couloir blanc où sont d'abord exposés des objets illustrant le concept de «wa» 和, donné comme une " conception traditionnelle de l'harmonie »: ils sont présentés comme «synthétisant des propriétés antagonistes ». Sous cette désignation se trouvent, entre autres, un tonneau à sake, un humidificateur d'air en forme de bulle ou un piano électrique.

Objets du premier couloir de l'exposition Wa de la MCJP, 2008.

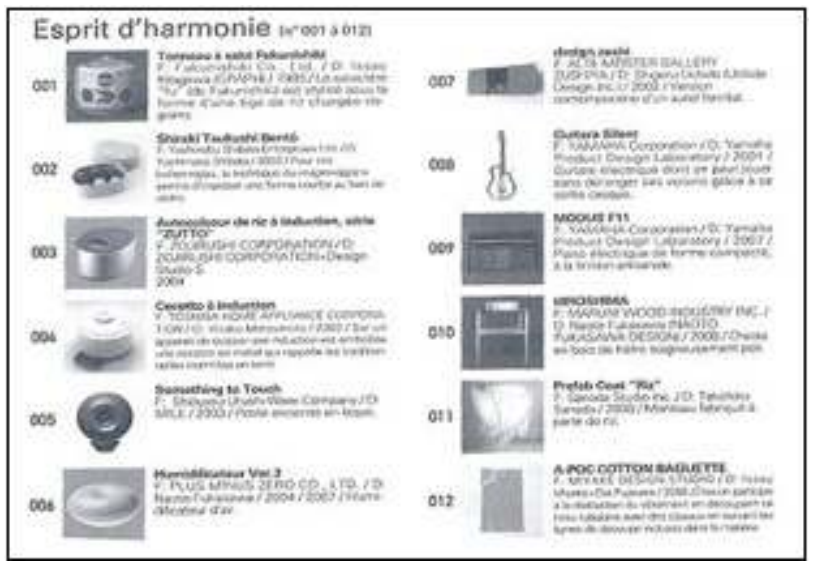

(PHOTO DE L'AUTEUR)

16 Ce couloir mène à une vaste salle sombre, aux murs tendus de toile noire, et décorée de pans de tissu blanc suspendus au plafond. Cent soixante pièces y sont annoncées par le dépliant comme constituant un panorama représentatif des objets utilisés au quotidien par les Japonais d'aujourd'hui. La date de création de la majorité de ces objets se situe entre 1980 et 2008, même si quelques pièces célèbres plus anciennes, tel le tabouret Butterfly, déjà vu au quai Branly, sont présentes.

Les objets sont installés dans des vitrines basses d'environ $1,20 \mathrm{~m}$ de haut, dont la largeur occupe presque tout l'espace, et qui forment des couloirs dans lesquels 
circulent les visiteurs. Les six premières rangées, et deux estrades au fond, présentent des objets regroupés selon des catégories fonctionnelles. Ces douze catégories sont dans l'ordre : «art de la table », "articles pour le bain », "électroménager et petit matériel électronique », «technologies numériques», «jouets», "papeterie», " articles divers pour la maison ", "vêtements et accessoires ", " emballages et sacs », "véhicules", «mobilier" et enfin, "luminaires". Les trois dernières vitrines présentent des objets répartis selon six «mots-clés » donnés en alphabet romain puis traduits : kawaii (mignon), kurafuto (craft), kime (finesse de grain), tezawari (sensation tactile), minimaru (minimal), kokoro kubari (prévenance). Ces mots renvoient d'après la brochure à « des concepts, des sensations ou des goûts particulièrement présents dans le design japonais ». Les catégories fonctionnelles, aussi bien que les concepts, sont introduits par de très courts textes qui figurent aux extrémités des vitrines. Par exemple, le paragraphe sur la notion de kokoro kubari débute ainsi : «Aux yeux des Japonais, la prévenance est une vertu, et aussi une forme de sagesse permettant de vivre harmonieusement et agréablement en société.» Enfin, en retrait de la salle principale, un "espace découverte » propose aux visiteurs de manipuler une dizaine d'objets, parmi lesquels une guitare, des cahiers ou une corbeille.

L'exposition est caractérisée par une volonté de proposer un panel très étendu qui soit « représentatif de la multitude d'objets » que les Japonais utilisent chaque jour, d'après les termes employés sur la brochure. Cela aboutit à la coexistence, dans la même salle, d'instruments de musique, de maquettes figurant des voitures, de stylos et d'une combinaison de natation pour la compétition. Certains objets sont signés par des designers célèbres (telle la chaise «Hiroshima» de Fukasawa Naoto), d'autres proviennent d'agences moins connues : l'assemblage apparaît hétéroclite.

Une partie des « visuels pour la presse » de l'exposition Wa de la MCJP

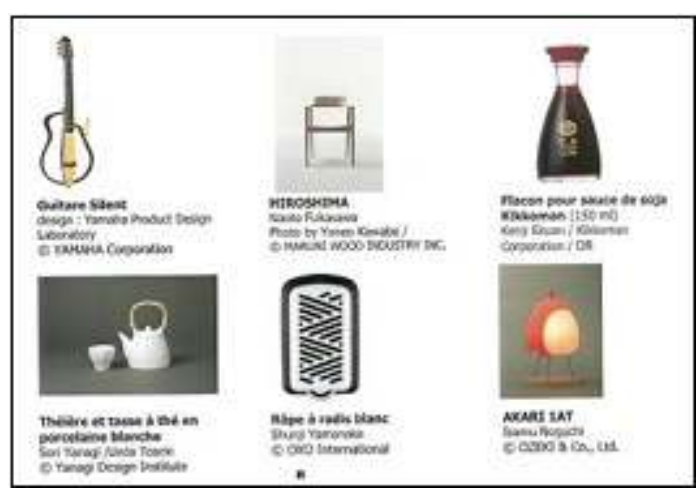

Trois des six objets présentés ici ont trait à l'alimentation.

(PHOTO DE L'AUTEUR)

\section{Les Arts décoratifs et la sensibilité Kansei}

Le Musée des Arts décoratifs accueille régulièrement des expositions dédiées au design. L'exposition Kansei, élaborée par le $\mathrm{METI}^{3}$ et le JETRO ${ }^{4}$, a réuni, d'après la brochure, « les secteurs privés, publics et universitaires dans un même effort de compétitivité industrielle». L'ambition de cette exposition est donc de promouvoir sur un plan commercial la production japonaise en matière de design. 
20 Au sein de la nef des Arts décoratifs, l'espace d'exposition Kansei est divisé en trois parties. Au centre, une grande salle est occupée par neuf écrans à LED qui diffusent des scènes animées, tirées de rouleaux illustrés du Roman du Genji. Deux sculptures monumentales occupent l'espace jusqu'au plafond: "Ikebana ", réalisée par l'école d'arrangement floral Ikenobō, est une structure exécutée à partir de baguettes ; tandis que «Scène de Nō» est une installation amovible réalisée par le designer Kita Toshiyuki. De grandes bannières portant la calligraphie des idéogrammes « kansei » 感 性donnent la traduction du mot (sensibilité), achevant de donner au lieu un caractère impressionnant.

21 Le couloir à gauche de la nef propose une exposition de cent quatre objets, posés sans vitrine sur des estrades blanches à diverses hauteurs. Les objets sont regroupés en trois thèmes, «l'expression ", "le geste » et «le cœur ", qui illustrent des "valeurs kansei » et renvoient chacun à quatre concepts différents. Tous ces mots sont exprimés en japonais, en kanji ou en hiragana, puis transcrits en alphabet, et enfin traduits : «動作, dōsa, le geste » explique par exemple un des panneaux ${ }^{5}$.

Comme à la MCJP, les objets sont d'usages et de valeurs extrêmement variés : des élastiques en forme d'animaux aux canettes de soda, en passant par le mobilier, la vaisselle, les bijoux et les téléphones portables, l'exposition couvre toutes sortes d'« objets du quotidien ». Ils sont tous de fabrication très récente (des années 2000), mais on note la présence de certains modèles dits «traditionnels » (bouilloire, fouets pour la cérémonie du thé...), qui ne sont pas des créations du design contemporain.

Le couloir opposé est fractionné en six salles : quatre d'entre elles présentent chacune un "maitre » artisan (en imprimerie, maroquinerie ou teinturerie) et ses productions, les deux autres sont réservées aux travaux de graphistes japonais. Certains des créateurs sont présents dans les salles.

Ici encore, l'exposition présente des éléments très divers. Les objets du premier couloir sont au moins aussi hétéroclites que ceux de la MCJP; la présence d'installations artistiques, ainsi que la présentation du travail d'ateliers artisanaux contribuent à accentuer la diversité du contenu exposé.

\section{Des registres d'objets mélangés}

25 La cohérence d'ensemble de chacune des expositions n'apparaît pas d'emblée. Or, rappelons-le, toute exposition construit un propos: quelle vision du Japon les organisateurs ont-ils voulu donner en sélectionnant ces objets de registres si différents ? Est-il possible de dégager ce qui les rassemble?

Les objets de l'exposition de la MCJP tenus pour représentatifs de ce qu'utilisent les Japonais aujourd'hui ne sont en réalité pas tous d'un usage domestique et quotidien. Parmi les articles de vaisselle, les téléphones portables, les cahiers d'écolier et les luminaires, on trouve un tonneau à sake, un tenori-on (instrument de musique numérique), ou même la reproduction d'un parc (Moerenuma, à Sapporo) conçu par le sculpteur Isamu Noguchi : il comprend plusieurs structures de divertissement et est représenté ici par des photographies dans la catégorie « jouets ».

Dans l'exposition Kansei, des chaises côtoient des montres, un vélo, des bouteilles en plastique, un robot thérapeutique destiné aux personnes malades, une guitare à quatre cordes... tous de fabrication très récente. L'intention est de montrer la vitalité du 
design moderne, mais l'ajout d'un fouet en bambou pour le thé, d'un éventail ou d'amulettes shintō, introduit également une dimension qui se veut « traditionnelle ».

Le Musée du quai Branly juxtapose également des objets de provenances et d'époques variées. Le mode de présentation fond les objets du passé et les objets contemporains sous une même étiquette, celle du mingei.

\section{Une scénographie muette}

L'impression de mélange des types d'objets est accentuée par la scénographie des expositions : en effet, la juxtaposition des objets ne s'accompagne que de très peu d'éléments explicatifs, et lorsqu'ils sont présents, ils sont d'accès difficile.

À la MCJP, peu d'explications sont fournies au visiteur. Mises à part quelques phrases à l'entrée sous le chapeau "Wa: harmonie », et les courts cartels qui introduisent les catégories, aucune indication n'est donnée dans les vitrines sur la fonction ou l'origine des objets. Seul figure un numéro, qui renvoie à la brochure, distribuée à l'entrée, laquelle comporte davantage d'explications. Cependant, il s'agit d'un document de $40 \mathrm{x}$ $60 \mathrm{~cm}$, plié en huit, qui reproduit les photographies des cent soixante objets avec pour chacun d'eux le nom de l'objet, sa date de création, le nom de son créateur et sa fonction. La police de caractères est très petite, l'impression, dans des dégradés de bleu ; l'ordre des objets est parfois différent de celui de l'espace d'exposition. Autant dire que dans la salle, qui est sombre, la lecture de la brochure est assez malaisée. De plus, une certaine confusion semble régner quant à la délimitation de l'espace muséographique : certaines pièces sont exposées à l'air libre, notamment un four et une machine à laver, et sont fréquemment manipulées par les visiteurs, lesquels sont vite rappelés à l'ordre par les gardiens.

31 Au Musée du quai Branly, la signalétique n'est pas plus explicite. Rappelons que les objets sont mis sur le même plan, sur un fond blanc, et sous un éclairage neutre. Ils sont placés à différentes hauteurs dans les vitrines. Les vignettes qui mentionnent leur nom, leur date de création et leur origine sont très sommaires, et posées au pied de la vitrine. Elles comportent des numéros, qui sont repris sur un schéma au sol, au centre de la vitrine, lequel reproduit la disposition des objets, afin de faire correspondre objets et vignettes. Ce système rend l'information qui figure sur les vignettes, déjà succincte, encore plus discrète et difficile d'accès: il faut se baisser pour pouvoir lire. Les différences d'époque et d'origine géographique, ou bien de démarche (conservation, création) n'apparaissent pas. Trois très petits écrans de dix pouces où défilent des photographies complètent la scénographie. Quelques paragraphes sur la vie et les positions intellectuelles de Yanagi Sōetsu figurent sur des panneaux, mais leur contenu est repris presque intégralement dans la petite brochure de quatre pages au format A 5 . Ces pages comportent également une interview de Germain Viatte, laquelle est le seul texte qui replace le mouvement des Arts populaires dans une perspective historique, de la modernisation de Meiji aux années 1930. Aucune information n'est donnée sur l'usage des objets.

32 Par contraste, au Musée des Arts décoratifs, la scénographie du premier couloir consacré aux objets donne une impression de luxe. Les éclairages sont nombreux, les éléments sur lesquels sont disposés les objets à l'air libre sont en noir et blanc brillant. De nombreuses calligraphies habillent l'espace, en blanc sur des panneaux noirs, qui mettent en avant et expliquent les concepts utilisés. Chaque objet est numéroté, 
possède un titre en japonais, une date et une provenance. Cependant, l'usage des objets n'est pas expliqué en salle. Il faut se reporter à la brochure : très fournie, elle comporte 53 pages et reprend l'intégralité des textes de l'exposition, avec en outre des textes signés par les organisateurs. Pour chaque objet, une photographie en couleurs accompagne un paragraphe expliquant l'usage et le rapport au concept qui lui est associé. La densité des informations fournies pour chaque objet dans le livret est telle qu'il est peu probable que le visiteur s'y reporte de façon systématique.

Description des élastiques en forme d'animaux

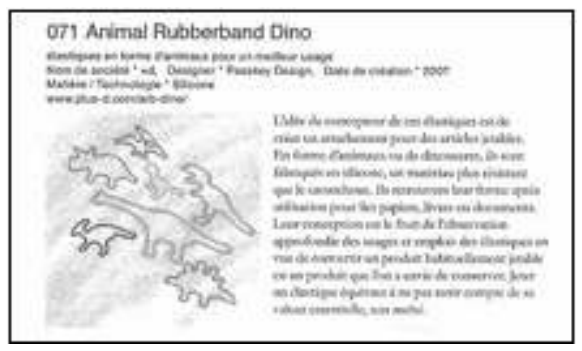

(cet objet est présenté dans la catégorie de la valeur mottai). Exposition Kansei, Musée des Arts décoratifs, 2008.

(PHOTO DE L'AUTEUR)

Par ailleurs, l'écriture japonaise, qu'il s'agisse des syllabaires ou des idéogrammes, est omniprésente dans les trois expositions, et particulièrement dans celle des Arts décoratifs. Les "concepts" qui définissent les objets sont calligraphiés dans la brochure, ainsi que sur de grands panneaux verticaux suspendus. Il y a là un procédé récurrent de présentation : chaque objet est associé à un concept en japonais, lequel est calligraphié, puis transcrit en alphabet romain, avant d'être éventuellement traduit, et, enfin, viennent les explications.

Présentation de la « valeur » karoyaka (la brochure l'associe au concept de kokoro).

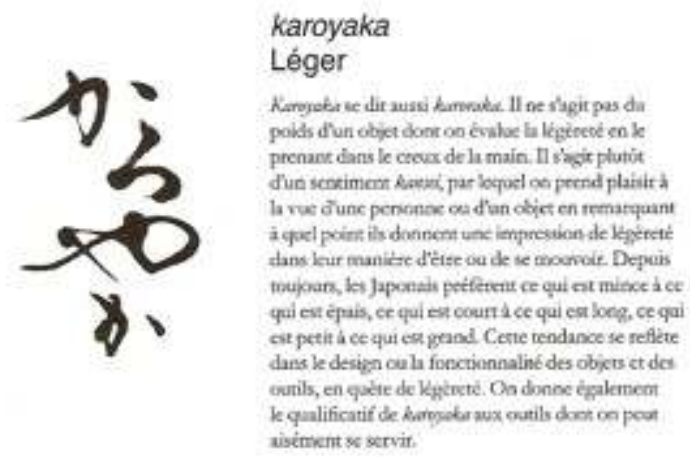

EXPOSITION KANSEI, MUSÉE DES ARTS DÉCORATIFS, 2008.

(PHOTO DE L'AUTEUR)

Or l'écriture japonaise est incompréhensible pour la très grande majorité des visiteurs français. Il s'agit pour beaucoup d'une écriture « muette », qui fait davantage obstacle à la compréhension qu'elle n'apporte des informations supplémentaires. Son rôle est surtout de donner une unité visuelle aux expositions.

La confusion des registres d'objets et le peu d'informations accessibles contribuent à donner un sentiment d'uniformité des expositions. Ce sentiment est soutenu par la 
rhétorique employée : il est chaque fois fait référence à l'ensemble des Japonais; il y est question de façon récurrente d'un "esprit » qui les caractériserait. Le communiqué de presse de la MCJP mentionne l'harmonie comme " une vertu japonaise par excellence ", et donc commune à tous les Japonais, qui gouverne l'ensemble de la production de design nipponne. L'ambition de la MCJP est de montrer ce qu'utilisent « les Japonais » quotidiennement. Dans le cas des Arts décoratifs, ce phénomène est particulièrement évident: le kansei est par exemple matérialisé par la sculpture scène de nō qui manifesterait «cette sensibilité des Japonais qui ressentent intimement cet espace comme une enceinte sacrée ». Les concepts énumérés par la même exposition sont formulés de façon encore plus emphatique, et insistent sur le fait qu'il s'agit de notions partagées par tous les Japonais. Par exemple, le mot hyōjō 表情est présenté de manière alambiquée comme « ce que les Japonais appellent l'« expression » d'un objet ».

Regardons concrètement à présent les objets et leur mise en valeur : comment ce sentiment d'unité est-il induit? Quels en sont les indices?

\section{À la recherche d'une unité de propos}

\section{Des unités partielles : les matériaux des objets}

37 Tout d'abord, l'unité des objets est obtenue par la matière dont ils sont faits. L'espace réservé aux objets en bambou dans l'exposition Mingei est révélateur. Il comporte des pièces de mobilier, notamment des tabourets, et mêle des objets anonymes de l'époque d'Edo, achetés par Charlotte Perriand, à des créations de Bruno Taut. L'unité de l'espace est donc créée par le bambou qui « semble incarner pour l'étranger le matériau type du Japon $»^{6}$, et fait l'unité des objets par sa qualité japonaise même. La japonité des objets des deux autres expositions semble, de la même façon, tenir dans le matériau qui les constitue. Par exemple, on trouve à la MCJP un manteau "fabriqué à partir de riz », une enceinte en laque, une corbeille en laque sur inox, de la vaisselle réalisée à l'aide de tissus de kimono moulés dans de la résine ; aux Arts décoratifs, un sac fait en fibre de riz, un téléphone en laque, des lanternes en papier... autant de matériaux naturels qui évoquent le Japon. Ceci se retrouve également au niveau de la scénographie: les panneaux explicatifs de l'exposition Mingei semblent faits de papier washi. Les pans de tissu accrochés au plafond de la salle de la MCJP ont également été conçus pour ressembler à ce type de papier.

La japonité semble aussi transparaitre dans l'usage des objets liés à l'alimentation. La prédominance des objets appartenant à ce registre est frappante : bols, flacons à sauce soja, autocuiseurs de riz, théières, couteaux de cuisine, râpe à radis blanc, fouet à thé, coupes à sake, couverts, baguettes... Ce qui fait l'identité du Japon semble être lié à ce que les Japonais mangent et à leur façon de manger. Ainsi, tout objet relevant de l'alimentaire est, de façon privilégiée, le support du discours des expositions (voir supra. Objets du premier couloir de l'exposition Wa de la MCJP, 2008. et Une partie des « visuels pour la presse » de l'exposition Wa de la MCJP )

À travers les matériaux mobilisés qui créent des unités partielles, et les références à la nourriture, l'identité japonaise des objets est façonnée par des éléments faisant référence à la nature; en d'autres mots, leur identité est présentée comme étant naturelle. 


\section{Des références partagées pour un « esprit » japonais}

40 l'argument d'un Japon atemporel, le recours à la langue japonaise comme véhicule de concepts spécifiques, ou la mise en avant de l'harmonie comme valeur à l'origine de la qualité du design japonais. Tout ceci fait référence à un " esprit », dont le lieu semble être le «cœur» japonais, le kokoro 心. Dans les brochures et les communiqués de presse, on observe une réelle convergence des discours autour de ces thématiques.

\section{L'atemporalité de la culture japonaise}

$41 \mathrm{Au}$ Musée du quai Branly, cette atemporalité est marquée, dès l'entrée, par la juxtaposition d'objets plus ou moins anciens. Ainsi présenté, le mouvement des Arts populaires apparait comme s'inspirant d'un « esprit » qui aurait traversé les siècles, et qui se donnerait à voir dans les objets issus de la culture japonaise, toutes époques confondues. C'est d'ailleurs le sentiment suscité par l'utilisation du terme «redécouverte » afin de qualifier l'entreprise de valorisation de l'artisanat populaire menée par Yanagi Sōetsu : l'emploi de ce mot suggère que ce dernier n'aurait fait que remettre sur le devant de la scène un caractère ("spirituel » en l'occurrence - d'après les textes de la brochure) qui aurait été propre de tout temps à ces objets, et qui aurait été oublié. Enfin, l'espace réservé à Charlotte Perriand met l'accent sur la continuité entre artisanat et design, s'appuyant sur les propos de cette dernière au sujet des objets mingei : « l'esprit de vérité qui a présidé à ces œuvres est un esprit éternel »7.

À la MCJP également, la récurrence d'expressions telles que "aujourd'hui comme hier », « depuis longtemps » ou «traditionnel » est frappante : elles sont présentes sur chaque cartel d'explication - bien que ces derniers soient succincts - et insistent sur la continuité entre passé et présent. Les objets et leurs procédés de fabrication « conservent en eux quelque chose de cet artisanat d'autrefois ». Le concept central de l'exposition est celui de " $w a »$, «l'harmonie». La légitimité de ce concept provient de son caractère très ancien, d'autant que ce mot est souvent utilisé pour désigner le Japon, comme dans les mots washi (papier japonais) ou wakotoba (sic, mots japonais) ${ }^{8}$ : le communiqué de presse rappelle ainsi qu'on le trouve dès 604 dans la première «Constitution » de Shōtoku Taishi. Le design moderne est défini par la formule : «la fabrication d'objets au Japon : quand le passé s'introduit dans le futur ». Enfin, toujours d'après ce communiqué, la scénographie établie par l'agence japonaise Tonerico "perpétue la tradition" de la maison japonaise, en proposant une "architecture minimaliste de l'espace $»^{9}$.

L'exposition des Arts décoratifs, quant à elle, présente le kansei comme une notion née avec le Roman du Genji en l'an mil et qui, traversant les siècles de façon immuable, s'actualise en tant que «faisceau de traditions japonaises façonnées au cours du temps » dans le design d'aujourd'hui : c'est ce qui justifie la présence dans un même espace des illustrations de rouleaux enluminés et d'objets des années 2000. Ces scènes présentées sur un support technologique moderne (de grands écrans à LED) montrent des images animées pixellisées, dans un style évoquant de vieilles pellicules de cinéma. Sur les panneaux et les brochures, le style de calligraphie utilisé est le style reisho, archaïsant et peu courant. L'anachronisme des effets visuels vise à renforcer le propos : il s'agit de montrer le kansei comme "transmis depuis cette époque", et qui, "de 
génération en génération, s'est perpétué jusqu'à aujourd'hui ». Le caractère immuable de la japonité apparait donc comme l'un des points centraux sur lesquels les trois expositions s'accordent.

\section{La langue japonaise comme clé}

Le recours à la langue japonaise est également un point commun aux trois expositions qui mettent en avant des concepts présentés comme spécifiquement japonais et difficilement traduisibles. Cela commence par les titres des trois expositions qui comportent des mots japonais, suivis d'un double point (:), lequel introduit une explication qui n'est pas nécessairement, ou pas seulement, la traduction exacte du terme japonais. L'intitulé de l'exposition est donc présenté d'emblée comme intraduisible. La brochure de l'exposition de la MCJP annonce que «tout comme les mots, les objets sont révélateurs de notre sensibilité ", afin d'introduire la classification des objets en six concepts "japonais $\aleph^{10}$. De la même façon, aux Arts décoratifs, les mots-clés traduisant la sensibilité « kansei » du design seraient des notions purement japonaises, qui s'expriment elles-mêmes par « des mots propres à la culture et de la vie quotidienne des Japonais ». Ils sont particulièrement abondants : les quatre concepts associés aux trois thèmes forment un total de quinze mots japonais, comme autant de clés de compréhension du design nippon.

Tableau 1 : classification des objets de l'exposition Kansei en thèmes et mots-clés

\begin{tabular}{|c|c|c|c|}
\hline $\begin{array}{l}\text { Thèmes } \\
\text { principaux }\end{array}$ & $\begin{array}{l}\text { 表情 } h y \overline{o j} \bar{o} \\
\text { L'expression }\end{array}$ & $\begin{array}{l}\text { 動作 dōsa } \\
\text { Le geste }\end{array}$ & $\begin{array}{l}\text { L kokoro } \\
\text { Le coeur }\end{array}$ \\
\hline \multirow[t]{4}{*}{$\begin{array}{l}\text { Mots- } \\
\text { clés } \\
\text { associés } \\
\text { aux thèmes }\end{array}$} & \begin{tabular}{l}
\multicolumn{2}{c}{ カ3げろ } \\
Kagerou \\
Passer de \\
l'ombre à la \\
lumière
\end{tabular} & $\begin{array}{l}\text { Uつえる } \\
\text { Shitsuraeru } \\
\text { Aménager } \\
\text { 1'espace }\end{array}$ & $\begin{array}{l}\text { もっtい } \\
\text { Mottai } \\
\text { La valeur } \\
\text { essentielle }\end{array}$ \\
\hline & $\begin{array}{l}\text { にしき } \\
\text { Nishiki } \\
\text { Le brocart }\end{array}$ & $\begin{array}{l}\text { Lぬる } \\
\text { Shinaru } \\
\text { Fléchir }\end{array}$ & $\begin{array}{l}\text { もてなし } \\
\text { Motenashi } \\
\text { L'accueil }\end{array}$ \\
\hline & $\begin{array}{l}\text { だーずまい } \\
\text { Tatazumai } \\
\text { L'allure }\end{array}$ & $\begin{array}{l}\text { はぬみ゙く } \\
\text { Habuku } \\
\text { Éliminer } \\
\text { superflu }\end{array}$ & $\begin{array}{l}\text { わらめळ } \\
\text { Karoyaka } \\
\text { Léger }\end{array}$ \\
\hline & $\begin{array}{l}\text { きぬ } \\
\text { Kime } \\
\text { Le grain }\end{array}$ & $\begin{array}{l}\text { おる } \\
\text { Oru } \\
\text { Plier }\end{array}$ & $\begin{array}{l}\text { むुवें } \\
\text { Musubi } \\
\text { Le noud }\end{array}$ \\
\hline
\end{tabular}

Cette exposition insiste encore sur le fait que les concepts exprimant l'essence du design japonais se traduisent par des mots purement japonais : les «yamato kotoba [...] par opposition aux kango, mots sino-japonais empruntés à la langue chinoise classique ». 
L'usage de mots incompréhensibles pour le public induit une impression d'opacité des concepts mêmes. Les deux sont indissociables: le commissaire général de Kansei souhaite «qu'au terme de cette exposition, le public français ait fait la découverte de cette nouvelle approche du design japonais et acquis la connaissance d'un nouveau mot : kansei ». Le design est présenté comme relevant d'une sensibilité exclusivement japonaise, et sa compréhension ne peut donc passer que par l'emploi du mot japonais.

\section{Une image classique d'un Japon « spirituel »}

\section{$L^{\prime}$ « harmonie »}

47 Le design présenté par des concepts opaques aux non japonophones dessine l'image d'un Japon difficile à saisir: sensible et spirituel, sa compréhension ne peut être qu'intuitive. Cet « esprit » japonais qui transparaîtrait dans les objets est caractérisé par le recours au motif de "l'harmonie », wa.

L'harmonie est mise en valeur en tant que concept propre au Japon dans les trois expositions de design, et particulièrement dans celle de la MCJP, qui en a fait son titre. Elle le définit comme une des «valeurs les plus prisées par le peuple japonais ». Pour appuyer cette affirmation, le communiqué de presse mentionne, nous l'avons déjà vu, la phrase d'ouverture de la Constitution de 604: «le Wa, valeur éminemment respectable, repose sur un principe, qui est d'éviter toute discorde. » Cette formule historique se réfère à une notion d'harmonie sociale ; or, elle est ici amalgamée à une idée d'harmonie esthétique. En effet, ce wa-là permettrait la fusion entre éléments anciens et modernes, ou encore entre industrie et artisanat, et serait à l'origine de la qualité propre du design japonais. La scénographie repose sur la même idée, d'après les organisateurs: épuré, en noir et blanc, l'espace d'exposition est "extrêmement japonais » en ce qu'il est caractérisé par « la beauté, l'ordre et l'harmonie ».

49 L'exposition des Arts décoratifs fait également référence à l'harmonie, «élément de la culture spirituelle et traditionnelle du Japon » et principe de fusion à l'origine de la spécificité du design japonais. Quant à celle du Musée du quai Branly, si elle n'insiste pas explicitement sur le terme, c'est encore l'idée de fusion et de symbiose entre l'artisanat populaire et le design moderne qui sous-tend son propos, et qui permet de faire figurer des œuvres de designers célèbres à côté d'objets populaires.

\section{Le « cœur » comme lieu d'une essence}

L'« esprit » que décrivent les trois expositions trouve son essence dans le « cœur » des Japonais. La notion de kokoro a été mise en valeur par les "études nationales" ou kokugaku au XvIII ${ }^{e}$ siècle. En réaction à l'influence chinoise, la recherche d'une essence première du Japon a conduit, au fil des relectures des textes du viII ${ }^{e}$ siècle comme le Kojiki ou le Man.yōshū, à l'émergence de ce concept qui relèverait d'une compréhension intuitive des choses, la compréhension rationnelle relevant de la logique chinoise.

51 Ce « cœur» est présent plus ou moins explicitement dans les trois expositions: on le trouve dans le «kokoro kubari » des objets de la MCJP, traduit par la «prévenance » du créateur envers l'utilisateur. Pour comprendre le design des objets, il s'agit, d'après la brochure de Kansei, de saisir « le mouvement qui se produit dans le geste ou le cœur $»^{11}$ de celui qui le crée : d'où le concept de dōsa, le «mouvement ». En manipulant l'objet, « il n'est pas rare que l'utilisateur commence à ressentir de l'émotion » : le « cœur » est 
un sentiment qui se transmet du producteur à l'utilisateur de l'objet. Pour décrire une lampe en forme de roseau, la brochure indique que "pour les Japonais, la vision des eulalies beige clair ondulant sous le vent évoque l'automne et donne un sentiment d'éphémère face au temps qui passe ». L'insistance de l'exposition Kansei à recourir aux yamato kotoba pour désigner "des représentations saisies par les cinq sens", et qui, d'après la brochure, ne s'écriraient qu'en syllabaire (hiragana) et non avec des caractères chinois, relève aussi de la logique du kokoro, dont la pureté est corrélée à son origine locale.

Si le kokoro dans ces trois expositions renvoie à des considérations esthétiques, il évoque également la dimension physiologique du cœur comme organe. Il est le lieu physique où se cristallise l'« esprit japonais ». Le Musée du quai Branly tient en effet, sur les pas de Yanagi Sōetsu, à « révéler la beauté des objets d'usage quotidien et leur dimension spirituelle ", laquelle donne à voir «l'esprit des peuples». Le kansei est " une riche sensibilité " sur laquelle l'exposition tente de "lever le voile», comme une "philosophie». La brochure de la MCJP présente encore cet esprit comme bien mystérieux : elle interroge « ce qui fait qu'au premier coup d'œil, un design est perçu, sans qu'on sache exactement pourquoi, comme spécifiquement japonais ».

\section{Resituer le discours des trois expositions}

Les expositions Wa et Kansei ont été réalisées par des Japonais ; quant à celle du Musée du quai Branly, bien que montée par une institution française, elle prend sa source dans l'institution japonaise qui lui a fourni les objets, accompagnés des discours qu'ils véhiculent. Le propos relayé par les trois expositions est, à l'arrivée, similaire et basé sur les mêmes arguments. Quelle place occupe ce discours dans le panorama des images diffusées par le Japon sur lui-même?

\section{L'empreinte des « nippologies »}

\section{Des références éprouvées}

De façon homogène, ces expositions diffusent l'idée d'un Japon anhistorique, d'une langue japonaise seule à même de véhiculer des concepts exclusivement japonais, d'un " esprit " japonais que caractériseraient les notions d'harmonie et de cœur. Or, ces références font écho aux thèses des «nippologies» (nihonjin-ron 日本人論, traduction empruntée à Philippe Pelletier ${ }^{12}$ ), dans lesquelles se retrouvent les mêmes éléments.

Travaux culturalistes produits par les Japonais sur eux-mêmes, les nippologies sont définies par Jacqueline Pigeot comme les « essais que les Japonais ont depuis la guerre consacrés à l'analyse de leurs particularités nationales, de leur société, de leur culture $»^{13}$. Le genre, identifié et étiqueté comme tel sur les étagères des libraires, est apparu après-guerre et a explosé à partir des années 1960 avec la haute croissance économique. Il est cependant à noter que des ouvrages parus dès le début $\mathrm{du} \mathrm{xx}^{\mathrm{e}}$ siècle s'y apparentent, tant dans le schéma formel que dans le contenu ${ }^{14}$.

Encore très vivante aujourd'hui, la catégorie recouvre des milliers de titres: Harumi Befu en a recensé des formes diverses qui se déploient dans un continuum entre travaux scientifiques et essais populaires: ouvrages, articles académiques, de magazines ou de journaux, ils constituent un phénomène culturel de masse ${ }^{15}$. Befu a 
également établi une typologie des principaux motifs autour desquels se cristallisent ces « traités sur les Japonais ». Or, il apparaît que ce sont précisément ceux que je viens de relever dans les trois expositions parisiennes.

Tout d'abord, l'anhistoricité de l'essence japonaise est au cœur des nippologies. Les heurts de l'Histoire sont passés sous silence. Pigeot cite ainsi des auteurs (Umesao Tadao, Nishio Kanji) dont la littérature " plaide pour l'immutabilité japonaise $»^{16}$.

En second lieu se dégage l'argument du langage, qui pour Befu " est inévitablement au cœur des nippologies» car les traits fondamentaux propres aux Japonais qui y sont énoncés sont exprimés en japonais par des concepts tenus pour difficiles à traduire ${ }^{17}$. Le moteur de l'argument de l'unicité par le langage est que le japonais n'est parlé que par les Japonais, et qu'il est parlé par tous les Japonais: c'est ce qu'il appelle l'« isomorphisme parfait » entre l'aire linguistique (les locuteurs de langue maternelle japonaise), la terre (les habitants du pays) et la culture. Ceci induit que la logique de la langue japonaise serait différente de celle des langues occidentales, et par là même, que le mode de pensée de ses locuteurs serait fondamentalement différent ${ }^{18}$.

Enfin, ce qui fait l'identité japonaise d'après les nippologies relève de l'intuition, et se trouve corrélée à l'emploi de motifs tels que l'harmonie ou le cœur. Pigeot qualifie l'harmonie de " dogme » autour duquel est organisée la structure sociale japonaise telle qu'elle est dépeinte par les nippologies ${ }^{19}$. Quant au kokoro comme lieu de l'essence de la culture, Befu le décrit comme une notion immanente, une "substance» de la quintessence japonaise, qui renvoie à un sentiment de nostalgie d'un Japon pur et premier $^{20}$.

Les expositions reprennent la rhétorique des nippologies, ce qui permet d'affirmer qu'elles s'inscrivent dans ce type de démarche. La structure des nippologies est régulière et propose des schémas-types de la culture nippone: un paradigme proprement japonais est dégagé (issu d'un registre écologique, linguistique, psychologique...) qui permet ensuite à l'auteur de décrire de nombreux aspects de la culture japonaise de façon «impressionniste» plus que sous la forme d'un développement dialectique ${ }^{21}$. Le même procédé est à l'œuvre dans les expositions, qui articulent des discours homogènes sur le Japon à partir de concepts érigés en paradigmes : le mingei au quai Branly, le wa à la MCJP, et le kansei aux Arts décoratifs.

\section{La méthode prescriptive}

61 L'homogénéité des expositions est annoncée comme étant une donnée émanant des objets présentés; pourtant, elle n'est que le fruit d'une scénographie et d'une rhétorique relevant des nippologies, qui font coexister des objets hétéroclites.

Cette rhétorique est entretenue par des formules standardisées et des arguments lourdement martelés. À la MCJP, l'«harmonie» se décline à chaque phrase; la rhétorique de la symbiose et de la fusion (des valeurs, des procédés de fabrication...) est le terreau de presque tous les articles rédigés par les commissaires et repris dans le communiqué de presse. Le Musée du quai Branly construit ses explications (de la scénographie et de la brochure) autour des champs sémantiques du populaire, de l'anonymat et du spirituel. Quant à Kansei, la quantité importante de textes appuyant le propos de l'exposition rend particulièrement frappant le procédé de standardisation des formules : la sensibilité, l'esprit, la nature, la tradition ou la philosophie sont autant de mots-clés qui se retrouvent à l'envi dans la brochure. Ils sont parfois associés au sein 
de tournures qui en deviennent absconses: par exemple, on l'a vu, dōsa est «le mouvement qui se produit dans le geste ou le cœur»; ou bien, «le sens esthétique [est] mûri au sein d'une culture profondément présente ». De plus, les paragraphes qui décrivent les objets exposés reprennent systématiquement le concept japonais qui leur est rattaché ; ils sont donc particulièrement répétitifs. Les brochures d'exposition ne sont apparemment pas conçues pour être lues de façon extensive par les visiteurs. Aussi, la répétition et la standardisation des formules servent-elles un souci aigu de communication: quel que soit le passage, l'essentiel du message contenu par l'exposition sera transmis.

Or, les expositions, et donc les textes qui les accompagnent - au moins dans le cas de Wa et Kansei - proviennent d'institutions japonaises. L'exposition des Arts décoratifs est explicitement organisée de bout en bout par le METI, et s'inscrit dans une démarche plus large de promotion industrielle à l'étranger, intitulée "Kansei et création de valeur ", initiée par le même ministère en mai 2007. Les Arts décoratifs ne semblent donc pas avoir pris part au processus d'élaboration de l'exposition parisienne. Quant à la MCJP, elle présente habituellement des expositions élaborées en interne à Paris, ou en partie en amont par la Fondation. Cependant, dans le cas de $W a$, le siège japonais a quasiment tout décidé, puisqu'il s'agit d'une "exposition itinérante " destinée à être présentée dans plusieurs pays étrangers. Les commissaires de l'exposition étaient donc tous Japonais et même la scénographie, qui est d'habitude laissée à une agence française, a été cette fois-ci réalisée par une agence nippone. Du choix des objets jusqu'à leur transport, en passant par le contact avec les entreprises les ayant produits, tout a été piloté par le siège tokyoïte de la Fondation. Les textes présentés dans l'exposition sont habituellement élaborés à partir d'une base en japonais fournie par le siège de la Fondation, qui laisse le soin à l'équipe en France de traduire, d'enrichir et d'adapter le contenu au public français. Or, cette fois-ci, la consigne a été de ne faire que traduire les textes de la signalétique, par ailleurs beaucoup plus courts qu'à l'accoutumée.

64 Tout ceci relève de la valeur "prescriptive » qui, selon Befu, caractérise le mode de fonctionnement des nihonjin-ron ${ }^{22}$. Befu postule en effet que les faits énumérés dans les nippologies et les théories qui en sont dégagées sont donnés comme des observations, alors qu'il s'agit d'un «idéal positif»: les Japonais décrits dans ces traités, et les caractères qui leur sont associés, deviennent des standards normatifs. C'est la même idée qu'énonce Pigeot lorsqu'elle affirme que les nippologies qui insistent sur le consensus social sont moins des «objets d'analyse» que des «formules conjuratoires $»^{23}$. Les institutions organisatrices des expositions en amont, au Japon, ont prescrit une vision du Japon à diffuser en France. Ainsi, la Fondation du Japon a formulé explicitement son désir de laisser les objets être "parlants ", afin de donner à voir un Japon intuitif, apparenté à celui des nippologies, et de l'imposer auprès du public parisien.

Au Musée du quai Branly, en revanche, si les objets proviennent du musée japonais, les commissaires insistent sur l'indépendance, tant au niveau financier qu'au niveau de la conception, dont ils ont bénéficié vis-à-vis du Mingeikan. Pourtant, nous l'avons vu, le propos est proche de celui des deux autres expositions. En cela, l'image renvoyée par le Japon en Occident apparait parfaitement assimilée. Cette appropriation illustre l'effet de miroir tendu par l'Autre dans la construction de l'identité japonaise. 


\section{Entre Japon et Occident, un jeu d'influences réciproques}

\section{L'identité japonaise : une construction en miroir}

Le discours d'identité culturelle produit par le Japon sur lui-même est donc installé et repris en France par les institutions exposantes qui le véhiculent à leur tour. Dans le cas du Musée du quai Branly, la démarche pourtant indépendante de l'institution japonaise aboutit à un propos balisé et marqué des mêmes arguments que les expositions élaborées au Japon. De plus, pour les trois expositions, ce propos est accepté et largement diffusé au grand public: ce qui s'observe notamment dans la presse qui a couvert les trois expositions. Les articles qui les évoquent reprennent les mots employés dans les communiqués de presse. Les formules sont parfois rigoureusement identiques, ce qui suggère un simple copié-collé de la source. Cependant, le discours développé plus en longueur dans le numéro spécial d'AD (Architectural Digest) consacré au design japonais montre une véritable récupération de la rhétorique des nippologies ${ }^{24}$ :

Et si l'essence du design se trouvait bel et bien au Japon? Sans que l'on sache exactement pourquoi, il nous arrive de distinguer, dans une myriade d'objets venus du monde entier, ceux issus du pays du Soleil Levant. Comme si, malgré la globalisation à outrance, l'œeil arrivait à reconnaître ce " petit quelque chose " d'éminemment nippon.

Sensibilité, compréhension intuitive, harmonie, atemporalité : les mêmes motifs sont repris, et dessinent les axes selon lesquels se bâtit l'image du Japon en France.

Précisément, l'identité japonaise que proposent les nippologies vaut pour et par sa diffusion à l'étranger. «Cette définition de soi ne vaut comme telle que parce qu'elle est acceptée par l'extérieur » : c'est pour cette raison, d'après Befu, que « le gouvernement japonais dépense tant d'énergie à propager les nippologies à l'étranger » en finançant leur traduction en anglais ${ }^{25}$, ou, ici, en exportant des expositions culturalistes.

Ici, les expositions Wa, Mingei et Kansei réactualisent un jeu de projections entre Japon et Occident qui leur est bien antérieur dans la définition de l'identité japonaise. Il s'agit d'un jeu de miroirs complexe, qui fonctionne par contact et réaction, dans les deux sens et sur une large échelle historique. L'image de soi donnée par le Japon s'est construite dans une confrontation permanente avec l'étranger. Michael Lucken l'a montré dans le cas de la formulation des concepts artistiques à la fin du XIX siècle $^{26}$; Laurence Caillet a également noté que les théories actuelles émises par des Japonais sur le Japon, pour être plébiscitées, doivent faire un détour par la recherche occidentale ${ }^{27}$. Ici, ce mouvement est illustré par un article rédigé par une commissaire de l'exposition du Musée du quai Branly, côté français, publié dans la revue Mingei, au Japon, afin de rendre compte de la réception de l'exposition en France ${ }^{28}$. Par ailleurs, elle attribue son succès à l'effort d'adaptation du contenu au public français. En d'autres termes, le Japon qui y a été présenté a fait mouche car il correspond déjà à une image très photogénique du Japon à laquelle le public français est sensible : le jeu de miroirs est sans fin.

\section{Rejet de l'étranger et ambition universaliste}

70 L'identité japonaise ainsi construite englobe deux tendances paradoxales et complémentaires. Parce qu'elle est purement japonaise, elle exclut les étrangers, qui ne peuvent la comprendre. Mais dans le même mouvement, parce qu'elle est spirituelle, 
l'essence japonaise a une vocation universelle : il s'agit de la diffuser le plus largement possible dans le monde.

Les nippologies sont toujours construites dans un rapport de comparaison et de différenciation, même implicite, à l'Occident. En effet, le discours véhiculé par les expositions se donne comme concernant l'ensemble de la population japonaise, et exclut les non Japonais de toute compréhension de cette identité. L'anecdote qu'on pouvait lire au Musée du quai Branly sur le cartel présentant les « objets en bambou » va clairement dans le sens d'une inaccessibilité des concepts japonais aux étrangers: Yanagi Sōetsu trouvait les objets achetés par Charlotte Perriand de très mauvais goût!

Le procédé d'exclusion des non Japonais est également récupéré lorsque les journalistes d'AD expliquent qu'il est nécessaire de passer par la «sémantique japonaise " pour tenter de comprendre la spécificité du design japonais. L'un d'eux juxtapose les concepts japonais sans les traduire, et ironise sur leur opacité («Kawaii, kurafuto, minimaru... rien que cela!»). Il écrit plus loin que le «kokoroburaki ${ }^{29}$ [est] une vertu proche de la sagesse ». Le terme japonais n'est pas employé par souci d'exactitude : les syllabes en sont négligemment mélangées. Le recours au concept japonais semble plutôt relever d'un goût pour l'exotique. Enfin, lorsqu'il conclut que "l'énigme de l'item nippon n'en reste pas moins à percer ", la différence insurmontable entre les Japonais et le reste du monde, argument premier des nippologies, est définitivement scellée dans son discours.

73 Cependant, cette essence purement japonaise est, plus encore, à vocation universelle. L'article de la revue Mingei cité plus haut affirme que la plus grande qualité de l'exposition a été de fasciner le public par le « discours universel » qui s'en dégageait: par la "parole silencieuse" des objets se dégageait une "vérité commune avec l'Occident ». Le texte signé par le ministre de l'Économie, du Commerce et de l'Industrie du Japon, en introduction de la brochure de l'exposition aux Arts décoratifs, présente la sensibilité kansei comme un concept que le Japon a "élucidé » et qu'il s'agit de " diffuser dans le monde ». Le propos prend ses racines dans un Japon essentialisé, mais il a une ambition internationale.

74 Lorsque le design japonais est présenté comme faisant la symbiose entre passé et présent, et entre artisanat et industrie, il s'érige en modèle. Il ne s'agit pas seulement de donner une image du Japon à voir à l'extérieur, mais de montrer également que les qualités de ce Japon sont un exemple à suivre, en l'occurrence, pour le design en général, d'où qu'il soit. De la même façon, la mise en avant des designers internationaux au sein de l'exposition Mingei montre que des étrangers se sont installés au Japon afin de puiser leur inspiration aux sources d'une essence spécifiquement japonaise, qui leur a permis d'élever leur création à un niveau transcendant les frontières, voire le temps : ce même "niveau plus élevé » qui est atteint grâce à la fusion des valeurs que permet le « wa».

Un article de Libération traitant de nos trois expositions soulève ces questions, en rapportant en guise d'introduction les propos du couturier Issey Miyake: «Il est possible d'envisager des mesures qui permettraient de refonder la nation sur un design ayant sa place dans le monde entier. $\aleph^{30}$ Le design semble être un support idéal pour les aspirations internationales de ceux qui veulent promouvoir une essence japonaise. 


\section{Conclusion}

$$
\text { propos de l'exposition Mingei en deux paragraphes: il évoque successivement la }
$$
propos de l'exposition Mingei en deux paragraphes: il évoque successivement la "dimension spirituelle» propre au Japon, puis l'ambition qu'il a eu de montrer "l'universalité des techniques " ${ }^{31}$. La juxtaposition des deux idées, dans cette synthèse, montre qu'elles forment l'armature minimale du propos de l'exposition: essence japonaise et universalisme. J'ai montré en quoi il s'agissait de deux tendances présentes dans les discours émis par les institutions exposantes et ceux qui les ont relayés. Mais de façon plus générale, le discours sur l'universalisme du modèle japonais semble être véritablement une part constituante des théories culturalistes.

Cette dynamique n'est cependant pas neutre : pour le philosophe Bernard Stevens, elle peut poser problème en ce qu'elle réactive la thématique du «dépassement de la modernité " par le Japon, laquelle porte une "coloration idéologique " qu'il juge dangereuse $^{32}$. En effet, il rappelle comment, dans les années 1930-1940, les philosophes de l'école de Kyōto en ont fait la justification de l'ultranationalisme et de l'impérialisme. La recherche de la spécificité japonaise s'accompagnait d'une "réinterprétation de la philosophie hégélienne de l'histoire », dans laquelle le Japon est placé en «terme ultime de l'avènement de l'Esprit ». La «vocation spirituelle» n'était alors qu'un prétexte, le projet universaliste étant au contraire tout à fait concret, puisqu'il s'agissait de remettre chaque nation à sa place, avec le Japon à leur tête. Le "dépassement de la modernité ", titre du célèbre colloque de 1942, s'exprime aujourd'hui sous la forme d'un "nouvel asiatisme »: ce qui le rend inquiétant est l'usage qui en est fait par les néo-nationalistes, pour lesquels la puissance économique du Japon est corrélée à la vocation universelle de la culture japonaise.

S'il semble donc nécessaire d'être prudent sur ses éventuelles implications politiques et idéologiques, Stevens concède que l'idée d'essence japonaise universelle reste néanmoins bonne à penser dans certains domaines, tels que l'esthétique. L'analyse des trois expositions Mingei, Wa et Kansei montre comment le design est un support privilégié dans le développement de cette rhétorique.

80 La culture, comme essence insaisissable, se veut ici incarnée dans des objets concrets. Retracer leur processus de sélection permet de mettre à jour la « vision du monde » qui les sous-tend, et qui les dépasse. La mise en perspective historique de Stevens vaut donc pour avertissement : la manipulation de l'identité / altérité japonaise décrite ici a des implications qui vont bien au-delà des portes des institutions muséales.

AD. Architectural Digest, $\mathrm{n}^{\circ} 79$, numéro spécial « Art, archi, photo, design... Inspiration Japon », novembre 2008, Paris, 194 p.

Cipango, 16 | 2009 


\section{BIBLIOGRAPHIE}

\section{Presse}

BoucRelle, Virginie, 2008, « Kansei, la nouvelle valeur du design », Planète Japon, nº 13.

FÈVRE, Anne-Marie, 4 novembre 2008, «France-Japon, relations d'harmonie ", Libération, http:// www.liberation.fr/culture/0101166925-france-japon-relations-d-harmonie (consultation :

27/11/2008).

GEOFFROY-SCHNEITER, Bérénice, novembre 2008, « Le Mingei ou la beauté dans l'ordinaire... », L'Eil, $\mathrm{n}^{\circ}$ 607, http://www.artclair.com/oeil/archives/docs_article/60031/le---mingei---ou-la-beautedans-l-ordinaire.php (consultation : 17/12/2009).

SHIRAHA Akemi 白羽明美, mars 2009, « Ke buranrī bijutsukan tenrankai hōkoku. Furansu, Pari tokubetsu-ten o oete»ケ・ブランリ一美術館展覧会報告ーフランス・パリ特別展を終えて (Compte rendu de l'exposition au Musée du quai Branly. Fin de l'exposition spéciale à Paris, France), Mingei 民藝, n675, mars 2009, p. 50-54.

\section{Communiqués de presse officiels}

Kansei, communiqué de presse en ligne : http://www.jetro.go.jp/france/topics/20081121308topics (dernière consultation : 4/12/08).

Wa : l'harmonie au quotidien : communiqué de presse papier.

\section{Catalogues d'exposition}

Fondation du Japon, $2008 \mathrm{Wa}$ : l'harmonie au quotidien. Design japonais d'aujourd'hui, catalogue d'exposition (Maison de la culture du Japon à Paris, Paris, 22 octobre 2008-31 janvier 2009), Tōkyō, Fondation du Japon, 165 p.

Musée du quai Branly, 2008 L'esprit mingei au Japon, catalogue d'exposition (Musée du quai Branly, Paris, 20 septembre 2008-11 janvier 2009), Paris, Musée du quai Branly / Actes Sud, 144 p.

\section{Dépliants distribués gratuitement}

L'esprit mingei au Japon, s. n., Musée du quai Branly, 2008, 4 p.

Wa : l'harmonie au quotidien, s. n., Maison de la culture du Japon à Paris, 2008, 8 p.

Kansei, Japan Design Exhibition, s. n., s. 1., 2008, 54 p.

\section{Matériaux secondaires}

BEFU Harumi, 2001 Hegemony of Homogeneity: an Anthropological Analysis of « Nihonjinron », Melbourne, Trans Pacific Press.

BENEDICT, Ruth, 1946, The Chrysanthemum and the Sword, Boston, Houghton Mifflin.

BuTEL, Jean-Michel, « \#005 Exposition : L'esprit Mingei au Japon - Musée du quai Branly », podcast, http://japethno.blogspot.com/ (dernière consultation 25/05/2009).

CAILlET, Laurence (éd.), 2006, Ateliers, n 30 : « Ethnographies japonaises », Département d'ethnologie de l'Université Paris X Nanterre.

CHAPPUIS, Romain, 2008, « La japonité selon Jeanne d'Arc », Critique internationale, $\mathrm{n}^{\circ}$ 38, p. 55-72. 
DAVALLON, Jean, 1999, L'exposition à l'œuvre. Stratégies de communication et médiation symbolique, Paris, L'Harmattan.

DoI Takeo 土井健郎, 1971, Amae no kōzō「甘え」の構造, Tōkyō, Kōbundō. Traduction française par E. Dale Saunders : Le jeu de l'indulgence. Étude de psychologie fondée sur le concept japonais d'amae, Paris, L'Asiathèque, 1982.

GONSETH, Marc-Olivier, HAINARD Jacques, KAERH Roland (éd.), 2002, Le musée cannibale, Neuchâtel, Musée d'ethnographie.

HOBSBAWN, Eric, RANGER Terence, 1983, The Invention of Tradition, Cambridge, Cambridge University Press.

IVY, Marilyn, 1995, Discourses of the Vanishing: Modernity, Phantasm, Japan, Chicago, University of Chicago Press.

L'ESTOILE, Benoît de, 2004, "Quand l'anthropologie s'expose », Critique. Revue générale des publications françaises et étrangères, $\mathrm{n}^{\circ}$ 680-681, vol. 60, jan-fév. 2004, p. 5-15.

L'ESTOILE, Benoît de, 2007, Le goût des autres. De l'Exposition coloniale aux arts premiers, Paris, Flammarion.

LUCKEN, Michael, 2001, L'art du Japon au vingtième siècle, Paris, Hermann.

OGUMA, Eiji, 小熊英二, 1995, 単一民俗神話の起源 Tan.itsu minzoku shinwa no kigen / The Myth of the Homogeneous Nation, Tōkyō, Shin.yōsha.

PELLETIER, Philippe, 2004, Japon - Idées reçues, Le cavalier bleu, 2004

PigEOT, Jacqueline, 1983, « Les Japonais peints par eux-mêmes », Le débat, nº 23, p. 19-33.

RAISON, Bertrand, 1989, L'empire des objets, Paris, éditions du May.

SABATA, Toyoyuki, 鯖田豊之, 1972 Nikushoku bunka to beishoku bunka 肉食文化と米食文化

(Civilisation carnivore et civilisation du riz), Tōkyō, Kōdansha.

STEVENS, Bernard, 1995, « Ambitions japonaises, nouvel asiatisme et dépassement de la modernité », Esprit, n² 213, juillet 1995, p. 5-29.

SUZUKI, Hideo, 鈴木秀夫, 1978, 森林の思考 - 砂漠の思考 Shinrin no shikō, sabaku no shikō (Pensée forestière, pensée désertique), Tōkyō, Nippon Hōsō Shuppan Kyōkai.

TIPTON, Elise K., CLARK John (éd.), 2000, Being Modern in Japan: Culture and Society from the 1910s to the 1930s, Honolulu, University of Hawai'i Press.

UMESAO, Tadao, 1983, Le Japon à l'ère planétaire, traduit par René Sieffert, Paris, Publications orientalistes de France.

UMESAO, Tadao, 2001, « Keynote Address: the Comparative Study of Collection and Representation ", in Umesao Tadao, Japanese Civilization in the Modern World, XVII, Collection and Representation, Senri Ethnological Studies, ${ }^{\circ}$ 54, p. 1-14.

VLASTOS, Stephen, 1998, Mirror of Modernity: Invented Traditions of Modern Japan, Berkeley, University of California Press.

YosHIDA, Kenji, 2001, «"Tōhaku” and "Minpaku” within the History of Modern Japanese Civilization: Museum Collections in Modern Japan », Japanese Civilization in the Modern World, XVII, Collection and Representation, Senri Ethnological Studies, n 54, p. 77-104. 


\section{NOTES}

1. Jean Davallon, L'exposition à l'œuvre. Stratégies de communication et médiation symbolique, Paris, L'Harmattan, 1999.

2. Benoît de L'Estoile, Le goût des autres. De l'Exposition coloniale aux arts premiers, Paris, Flammarion, 2007.

3. METI : Ministry of Economy, Trade and Industry (ministère japonais de l'Économie, du Commerce et de l'Industrie).

4. JETRO : Japan External Trade Organization (Organisation du commerce extérieur japonais).

5. Voir plus loin la liste de ces concepts et leurs traductions.

6. Citation tirée du texte du cartel figurant sur l'espace « les objets en bambou ».

7. Extrait du discours d'introduction pour une exposition au grand magasin Takashimaya à Tōkyō en 1941.

8. Le terme wakotoba est effectivement utilisé dans les documents de cette exposition, mais il n'est pas attesté en japonais - à moins qu'il ne s'agisse d'un néologisme créé pour l'occasion - et il s'agit sans doute d'une erreur du traducteur pour yamato kotoba qui s'écrit avec les mêmes caractères.

9. Selon les termes du communiqué de presse.

10. Pour mémoire : kawaii (mignon), kurafuto (craft), kime (finesse de grain), tezawari (sensation tactile), minimaru (minimal), kokoro kubari (prévenance).

11. Je reviendrai plus loin sur la tournure approximative utilisée ici.

12. Voir par exemple Japon - Idées reçues, Le cavalier bleu, 2004, p. 9-12.

13. Jacqueline Pigeot, "Les Japonais peints par eux-mêmes », Le débat, $n^{\circ} 23$, 1983, p. 19-33, ici p. 19.

14. Notamment Iki no kōzō de Kuki Shūzō, publié en 1930, dans lequel l'auteur définit une notion proprement japonaise, l'iki, donnée comme clé de l'esthétique traditionnelle; Kuki Shūzō, La structure de l'iki, Tōkyō, Maison franco-japonaise, 1984 (pour la traduction française).

15. Harumi Befu, Hegemony of Homogeneity: An Anthropological Analysis of "Nihonjinron", Melbourne, Trans Pacific Press, 2001.

16. Pigeot, op. cit., p. 23.

17. Befu, op. cit., p. 35.

18. Ibid., p. 36.

19. Pigeot, op. cit., p. 30.

20. Befu, op. cit., p. 32-33.

21. Par exemple, le nihonjin-ron de Suzuki Hideo oppose le paysage de forêt, typiquement japonais, au paysage désertique de l'Occident : le premier serait la cause de la capacité d'analyse aiguë propre aux Japonais, par contraste avec le second, qui déterminerait l'esprit de synthèse. Suzuki Hideo, Shinrin no shikō, sabaku no shikō (Pensée forestière, pensée désertique), Tōkyō, Nippon hōsō shuppan kyōkai, 1978, cité par Laurence Caillet, «Introduction », Ateliers, no 30 : «Ethnographies japonaises », Département d'ethnologie de l'Université Paris X Nanterre, 2006, p. 16.

22. Befu, op. cit., p. 78.

23. Pigeot, op. cit., p. 32.

24. Christian Simenc, «La beauté de l'utile », AD. Architectural Digest, $\mathrm{n}^{\circ} 79$, numéro spécial « Art, archi, photo, design... Inspiration Japon », novembre 2008, p. 68.

25. Befu, op. cit., p. 82.

26. Michael Lucken, L'art du Japon au vingtième siècle, Paris, Hermann, 2001, p. 23-27.

27. Laurence Caillet, op. cit., p. 9-34.

28. Shiraha Akemi 白羽明美, « Ke buranrī bijutsukan tenrankai hōkoku. Furansu, Pari tokubetsuten o oete»ケ・ブランリ一美術館展覧会報告 — フランス・パリ特別展を終えて (Compte 
rendu de l'exposition au Musée du quai Branly. Fin de l'exposition spéciale à Paris, France), Mingei 民藝, nº 675, mars 2009, p. 50-54.

29. Il s'agit en réalité de « kokoro kubari».

30. Anne-Marie Fèvre, "France-Japon, relations d'harmonie», Libération, 4 novembre 2008, consultable en ligne: http://www.liberation.fr/culture/0101166925-france-japon-relations-dharmonie (dernière consultation : 27/11/08).

31. Bérénice Geoffroy-Schneiter, "Le Mingei ou la beauté dans l'ordinaire... ", L'Æil, $\mathrm{n}^{\circ} 607$, novembre 2008, consulté en ligne: http://www.artclair.com/oeil/archives/docs_article/60031/ le---mingei---ou-la-beaute-dans-l-ordinaire.php (27/11/08).

32. Bernard Stevens, « Ambitions japonaises, nouvel asiatisme et dépassement de la modernité », Esprit, $\mathrm{n}^{\circ}$ 213, juillet 1995, p. 5-29.

\section{RÉSUMÉS}

En 2008-2009 ont été organisées à Paris trois expositions sur le design japonais, aux ambitions différentes. Elles font cependant toutes écho aux thèses des «nippologies » (nihonjinron) et insistent sur les spécificités japonaises.

In 2008-2009 were held in Paris three exhibitions on Japanese design. Although expectations were different, they referred to "Theories of Japaneseness" (nihonjinron) and a supposed particularism.

\section{INDEX}

Thèmes : anthropologie, ethnologie, histoire de l'art, muséolographie キーワード : minshū geijutsu 民衆芸術, taishū bunka 大衆文化, minzokugaku 民俗学, minzoku 民俗, mingei undō 民芸運動, bijutsukan 美術館, nihonjinron 日本人論, Yanagi Sōetsu 柳宗悦 (1889-1961), jinruigaku 人類学, minzokugaku 民族学, bijutsushi 美術史, hakubutsukan shiryō kiroku gijutsu 博物館資料記録技術, Shōwa jidai 昭和時代 (1923-1945), Shōwa jidai 昭和時代 (1945-1989)

Keywords : Anthropology, Ethnology, Folk Art, Material Culture, Mingei, Museography, Museum, nihonji-ron, Popular Culture

Mots-clés : arts populaires, culture populaire, études folkloriques, folklore, mingei mouvement artistique, musée, nihonji-ron, nippologie, Yanagi Sōetsu (1889-1961)

Index chronologique : Shōwa (1923-1945), Shōwa (1945-1989) 